\title{
Optimal Locations and Sizing of Capacitors for Voltage Stability Enhancement in Distribution Systems
}

\author{
Mohan. G \\ Reader in Electrical Engineering \\ Annamalai University \\ Annamalainagar - 608 002, Tamil \\ Nadu, India.
}

\author{
Aravindhababu. $\mathrm{P}$ \\ Professor of Electrical Engineering, \\ Annamalai University \\ Annamalainagar - 608 002, Tamil \\ Nadu, India.
}

\begin{abstract}
Voltage instability occurs in power systems when the system is unable to maintain an acceptable voltage profile under an increasing load demand and/or configuration changes. The operating conditions of the present day distribution systems are closer to the voltage stability boundaries due to the ever increasing load demand. This paper presents a new algorithm for optimal locations and sizing of static and/or switched shunt capacitors in order to enhance voltage stability in addition to improving the voltage profile and minimising losses. Test results on 33 and 69-node distribution systems reveal the superiority of this algorithm.
\end{abstract}

\section{Key words:}

voltage stability, radial distribution systems, capacitor placement.

\section{Nomenclature \\ between nodes $\mathrm{k}$ and $\mathrm{m}$ \\ nn \\ $r_{k m}+j x_{k m}$ 1 \\ $P_{1-m}+j Q_{1-m}$ \\ power load at node-m

CP
VM
VS
VSI
$\boldsymbol{L}_{m}$
$\boldsymbol{L}^{t}$
$\boldsymbol{L}^{\text {low }}$
system
PA
$\boldsymbol{P}_{k m}$

distribution line connected

the nodes beyond node-m plus the real power load of node-m itself plus the sum of the real power losses of all the branches beyond node-m.

$Q_{k m}$

sum of reactive power loads of all the nodes beyond node-m plus the reactive power load of

or placement

voltage stability index

VSI of line- $l$ or node- $m$

threshold value for VSI

lowest value of VSI in the

proposed algorithm

sum of real power loads of all node-m itself plus the sum of the reactive power losses of all the branches beyond node-m.

$Q_{m}$ reactive power delivered by node-m

$Q_{m}{ }^{o} \quad$ reactive power delivered by node-m before compensation

$Q_{L-\min \text { and }} Q_{L-\max }$ system minimum and maximum reactive power demands repectively

$\begin{array}{ll}Q c_{m} & \text { net } \\ \begin{array}{l}\text { compensation at node- } m \\ Q c_{m}{ }^{o}\end{array} & \text { initial value of } Q c_{m} \\ V_{k} & \text { voltage magnitude at node-k } \\ V & \text { lowest value of VM in the } \\ \text { system } & \text { voltage angle at node-k } \\ \delta_{k} & \delta_{k}-\delta_{m} \\ \delta_{k m} & L_{m}-L^{t}, \text { mismatch of VSI at } \\ \Delta L_{m} & \\ \text { node- } m & \text { additional reactive power } \\ \Delta Q_{m} & m\end{array}$

voltage stability

\section{INTRODUCTION}

Modern power systems are more heavily loaded than ever before to meet the growing demand and one of the major problems associated with such a stressed system is voltage collapse or voltage instability. Voltage collapse is characterized by a slow variation in system operating point due to increase in loads in such a way that the voltage magnitude gradually decreases until a sharp accelerated change occurs [1]. The problem of voltage collapse may simply be explained as the inability of the power system to supply the required reactive power or because of an excessive absorption of the reactive power by the system itself [2]. The problem of voltage instability or collapse has become a matter of great concern to the utilities in view of its prediction, prevention and necessary corrections to ensure stable operation. In recent years, the load demand in distribution 
systems are sharply increasing due to economical and environmental pressures. The operating conditions are thus closer to the voltage stability boundaries. In addition, distribution networks experience frequent distinct load changes. In certain industrial areas, it is observed that under certain critical loading conditions, the distribution system suffers from voltage collapse. In 1997, the voltage instability problem in a distribution system that spread to a corresponding transmission system caused a major blackout in S/SE Brazilian system [3]. Recently, the voltage stability (VS) of radial distribution system has been studied and various voltage stability indices have been developed [4-7].

Capacitors are commonly used to provide reactive power support in distribution systems. The amount of reactive compensation provided is very much linked to the placement of capacitors in distribution feeders in the sense that it essentially determines the location, size, number and type of capacitors to be placed, as it reduces power and energy losses, increases the available capacity of the feeders and improves the feeder voltage profile. Numerous capacitor placement (CP) methods with a view of minimising losses have been suggested in the literature [8-13]. Optimal allocation and sizing of capacitor banks for profitability and voltage enhancement of PV system on feeders has been suggested [14]. The effect of location and capacity of distributed generation on voltage stability of distribution systems has been studied [15]. Algorithms for enhancing voltage stability of transmission systems by optimal CP have been discussed [16-17]. A relationship between voltage stability and loss minimisation has been developed and the concept of maximising voltage stability through loss minimisation has been outlined [18-19]. Measures for enhancing voltage stability of distribution systems by network reconfiguration that alters the topological structure of the distribution feeders by rearranging the status of switches have been suggested [2023].

Though several attempts have been made to use capacitor banks for loss minimisation, voltage profile improvement, improvement of power factor, etc, hardly any work has been reported involving them with a view of enhancing voltage stability of distribution systems. The rapid growth of system size and exponentially increasing power demand at distribution level necessitate efficient and effective methodologies to avoid voltage collapse and the consequent occurrence of black-outs. This paper is thus directed towards enhancing VS of distribution systems through the use of capacitor banks.

A new algorithm that uses the VSI suggested in [7], for optimal locations and sizing of static and/or switched shunt capacitors in radial distribution system for voltage stability enhancement is proposed in this paper. This method improves voltage profile and reduces system losses in addition to enhancing voltage stability. The method is tested on 33 and 69-node radial distribution systems and the results are presented.

\section{Proposed CP Algorithm}

The aim of the present work is to place capacitor banks at optimal locations with a view to enhance voltage stability of radial distribution systems. The method uses VSI suggested in [7] and offers reactive power support at the appropriate nodes to improve VSI values towards a fixed threshold value, which is chosen based on system configuration and the operating state. The proposed algorithm (PA) determines the number, sizes, locations and types for capacitors to be placed on a distribution system in order to enhance voltage stability.

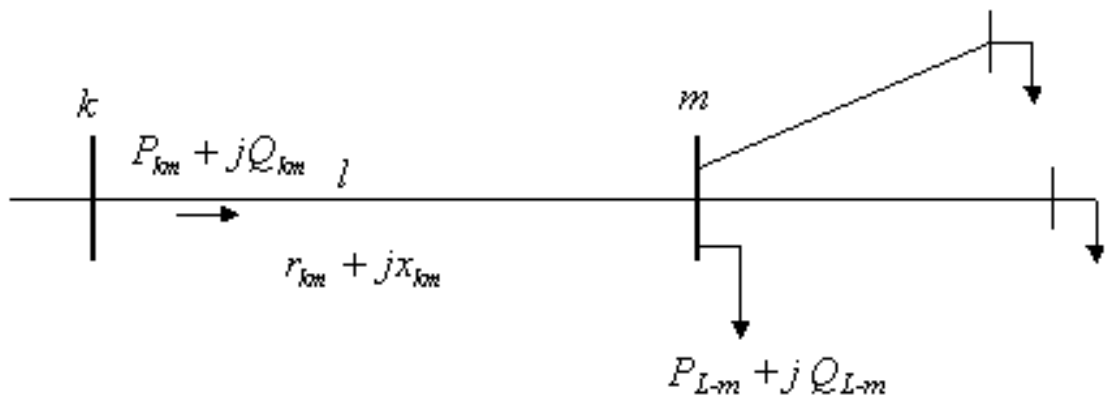

Fig. 1 Sample Distribution Line

The VSI, which varies between unity at no load and zero at voltage collapse point, for line-l or for node-m can be determined by

$$
L_{m}=\left[2 \frac{V_{m}}{V_{k}} \cos \left(\delta_{k}-\delta_{m}\right)-1\right]^{2}
$$

Linearising Eq. (1) and neglecting the higher order terms

$$
\Delta L_{m}=\frac{d L_{m}}{d V_{m}} \Delta V_{m}
$$


where

$$
\begin{aligned}
\Delta L_{m}=L_{m} & -L^{t} \\
& \frac{d L_{m}}{d V_{m}}=8\left[\frac{V_{m}}{V_{k}^{2}}\right] \cos ^{2} \delta_{k m}-4 \frac{\cos \delta_{k m}}{V_{k}}
\end{aligned}
$$

The net reactive power delivered by node- $\boldsymbol{m}_{\text {can be written as }}$

$$
Q_{m}=-Q_{m k}=-V_{m}^{2} B_{b m}-V_{m} V_{k} G_{k m} \sin \delta_{m k}+V_{m} V_{k} B_{b m} \cos \delta_{m k}
$$

Linearising Eq. (4) and neglecting the higher order terms

$$
\Delta Q_{m}=\frac{d Q_{m}}{d V_{m}} \Delta V_{m}
$$

where

$$
\frac{d Q_{m}}{d V_{m}}=-2 V_{m} B_{k m}-V_{k} G_{k m} \sin \delta_{m k}+V_{k} B_{k m} \cos \delta_{m k}
$$

Rearranging Eq. (2) and substituting it in Eq. (5),

$$
\Delta Q_{m}=\frac{d Q_{m}}{d V_{m}}\left[\frac{d L_{m}}{d V_{m}}\right]^{-1} \Delta L_{m}
$$

The VSI at all nodes are computed using Eq. (1). If all these values are greater than a fixed threshold value, it indicates that the system is away from the voltage instability point and the system does not require any reactive power compensation; else the nodes, whose VSI values are lower than the threshold value, are chosen as the candidate nodes for compensation. However, the node- $m$ having the lowest VSI value is chosen for $\mathrm{CP}$ and the additional reactive power compensation, $\triangle Q_{m}$, to be provided at this node can be obtained by solving Eq. (7). The calculated reactive power support is provided at node- $\boldsymbol{m}$ and the above process is continued till all the VSI values become less than the threshold value. The chosen node- $\boldsymbol{m}$ is said to be optimal as it is the most vulnerable node from voltage stability point of view and reactive support at that node ensures the system to

$$
\begin{aligned}
& \text { chosen to be fixed capacitors when } \\
& \left.\qquad Q_{L-\min } \leq \sum_{m=2}^{m} Q_{L-m} \leq Q_{L-\max }\right\}
\end{aligned}
$$

be far away from the voltage instability point when compared to providing reactive support at all other nodes one at a time.

The maximum compensation at each node is limited to the initial reactive power delivered by the respective node prior to compensation for avoiding over-dimensioning of the capacitor banks as,

$$
Q c_{m} \leq Q m^{\circ}
$$

The capacitor to be installed at a specific node may be either fixed or switched type, which is based on the system minimum and maximum reactive power demands, $Q_{L-\text { min }}$ and $Q_{L-\max }$ in a defined period. They are

$$
\left\{\sum_{m=2}^{m} Q_{L-m} \leq Q_{L-\min }\right\} \quad \text { and }{ }_{\text {switched }} \text { capacitors }
$$

The algorithm of the proposed method is summarized as follows:
1. Read the system data.

2. Choose a fixed threshold value, $\boldsymbol{L}^{t}$.

3. Set flag $=0$ and $Q c_{m}=0$ for all the nodes. 
4. $Q c_{m}{ }^{\circ}=Q_{C m}$ for all the nodes.

5. Carryout distribution power flow.

6. Compute VSI values, $\boldsymbol{L}_{m}$, at all nodes using Eq. (1).

7. Choose the node having lowest value of VSI, $L^{\text {low }}$, as the sensitive node- $m$ for CP.

8. If $L^{\text {low }} \geq \boldsymbol{L}^{t}$, or if flag $=\mathbf{1}$ for all the candidate nodes, then go to step (10).

9. Solve Eq. (7) for $\Delta Q_{m}$ and then compute the net compensation at node $-m$

$$
Q c_{m}=Q c_{m}{ }^{\circ}+\Delta Q_{m}
$$

10. Check for reactive power compensation limit:

11. If $Q c_{m} \geq Q_{m}{ }^{o}$, then $Q c_{m}=Q_{m}{ }^{o}$ and set flag $=1$ for node-m to avoid this node in the subsequent computations. and go to step (4).

12. The optimal locations for $\mathrm{CP}$ are obtained. Choose the nearest available value of capacitor from the computed values of $Q c_{m}$

13. Stop.

\section{Simulation}

The proposed algorithm is tested on 33 and 69-node distribution systems. The line and load data for these two systems are obtained from the references [24] and [22]. The power flow suggested in [25] is used in this study. The size of the capacitor banks considered in this study are 150, 300, 450, 600 and $900 \mathrm{kVAR}$. The results are obtained for light, medium, full and over load conditions by multiplying the base-load by a factor $0.5,0.8,1.0$ and 1.1 respectively. The threshold value for VSI is taken as 0.95 for both systems. The threshold value depends on the power system configuration and the operating state. If this value is fixed too low, it does not ensure that the power system will be maintained in a stable state. If this value is fixed too high, the reactive power to be provided will be too excessive.

\section{3 node test system:}

The minimum reactive power compensation required to enhance voltage stability for different loading conditions for 33 node system are given in Table-1. The system minimum and maximum reactive power demands are $1150 \mathrm{kVAR}$ and $2530 \mathrm{kVAR}$ respectively. The size and type of capacitor banks required for 33 node system based on the variation of reactive power demands are given in Table-2. Two fixed type of capacitor banks with a net rating of $1050 \mathrm{kVAR}$ are permanently connected at node- 6 and node- 8 to supply reactive power at all loading conditions. Switched capacitor banks ranging from $150 \mathrm{kVAR}$ to $300 \mathrm{kVAR}$ are connected, as shown in Table-2, to offer additional reactive power. Table-3 compares $\boldsymbol{L}^{\text {low }}, \boldsymbol{V}^{\text {low }}$ and system losses before and after $\mathrm{CP}$ for different
Table-1 Requirement of VAR compensation for 33 node system

\begin{tabular}{|c|c|c|}
\hline Load Level & Node-6 & Node-8 \\
\hline Light Load & --- & --- \\
\hline Medium Load & $450 \mathrm{kVAR}$ & --- \\
\hline Full load & $1200 \mathrm{kVAR}$ & $150 \mathrm{kVAR}$ \\
\hline Overload & $1350 \mathrm{kVAR}$ & $300 \mathrm{kVAR}$ \\
\hline
\end{tabular}

Table-2 Type and Size of Capacitor placed for 33 node system

\begin{tabular}{|c|c|c|c|}
\hline Type & $\begin{array}{c}\text { Size } \\
(\mathrm{kVAR})\end{array}$ & Node-6 & Node-8 \\
\hline \multirow{2}{*}{ Fixed } & 150 & & 1 No \\
\cline { 2 - 4 } & 900 & $1 \mathrm{No}$ & \\
\hline \multirow{2}{*}{ Switched } & 150 & $1 \mathrm{No}$ & $1 \mathrm{No}$ \\
\cline { 2 - 4 } & 300 & $1 \mathrm{No}$ & \\
\hline
\end{tabular}

Table-3 Performance of the PM for 33 node system

\begin{tabular}{|c|c|c|c|c|c|c|}
\hline \multirow{2}{*}{$\begin{array}{c}\text { Load } \\
\text { Level }\end{array}$} & \multicolumn{3}{|c|}{ Before CP } & \multicolumn{3}{c|}{ After CP } \\
\cline { 2 - 7 } & $L^{\text {lon }}$ & $V^{\text {lor }}$ & $\begin{array}{c}\text { Loss } \\
(\mathrm{kW}\end{array}$ & $L^{\text {lon }}$ & $V^{\text {lor }}$ & $\begin{array}{c}\text { Loss } \\
(\mathrm{kW}\end{array}$ \\
\hline Light & 0.96 & 0.95 & 48.7 & 0.97 & 0.96 & 38.0 \\
Load & 4 & 4 & 8 & 8 & 5 & 5 \\
\hline Medium & 0.94 & 0.92 & 130. & 0.96 & 0.93 & 101. \\
Load & 1 & 4 & 71 & 1 & 6 & 93 \\
\hline Full & 0.92 & 0.90 & 210. & 0.95 & 0.91 & 163. \\
load & 6 & 4 & 97 & 0 & 9 & 37 \\
\hline Overloa & 0.91 & 0.89 & 259. & 0.94 & 0.91 & 197. \\
d & 7 & 3 & 64 & 8 & 4 & 24 \\
\hline
\end{tabular}


Table-4 Requirement of VAR compensation for 69 node system

\begin{tabular}{|c|c|c|}
\hline Load Level & Node-57 & Node-58 \\
\hline Light Load & $450 \mathrm{kVAR}$ & --- \\
\hline Medium Load & $900 \mathrm{kVAR}$ & --- \\
\hline Full load & $1200 \mathrm{kVAR}$ & $1050 \mathrm{kVAR}$ \\
\hline Overload & $1350 \mathrm{kVAR}$ & $1350 \mathrm{kVAR}$ \\
\hline
\end{tabular}

Table-5 Type and Size of Capacitors placed for 69 node system

\begin{tabular}{|c|c|c|c|}
\hline Type & $\begin{array}{c}\text { Size } \\
(\mathrm{kVAR})\end{array}$ & Node-57 & Node-58 \\
\hline Fixed & 450 & & 1 No \\
& 900 & $1 \mathrm{No}$ & \\
\hline \multirow{2}{*}{ Switched } & 150 & $1 \mathrm{No}$ & \\
& 300 & $1 \mathrm{No}$ & $1 \mathrm{No}$ \\
& 600 & & $1 \mathrm{No}$ \\
\hline
\end{tabular}

Table-6 Performance of the PM for 69 node system

\begin{tabular}{|c|c|c|c|c|c|c|}
\hline \multirow{2}{*}{$\begin{array}{c}\text { Load } \\
\text { Level }\end{array}$} & \multicolumn{3}{|c|}{ Before CP } & \multicolumn{3}{c|}{ After CP } \\
\cline { 2 - 7 } & $L^{\text {low }}$ & $V^{\text {low }}$ & $\begin{array}{c}\text { Loss } \\
(\mathrm{kW})\end{array}$ & $L^{\text {low }}$ & $V^{\text {low }}$ & $\begin{array}{c}\text { Loss } \\
(\mathrm{kW})\end{array}$ \\
\hline $\begin{array}{c}\text { Light } \\
\text { Load }\end{array}$ & 0.942 & 0.942 & 70.20 & 0.967 & 0.961 & 64.19 \\
\hline $\begin{array}{c}\text { Medium } \\
\text { Load }\end{array}$ & 0.903 & 0.903 & $\begin{array}{c}192.8 \\
8\end{array}$ & 0.930 & 0.924 & $\begin{array}{c}143.6 \\
1\end{array}$ \\
\hline \begin{tabular}{c} 
Full load \\
\hline
\end{tabular} & 0.874 & 0.875 & $\begin{array}{c}317.7 \\
3\end{array}$ & 0.925 & 0.911 & $\begin{array}{c}247.9 \\
6\end{array}$ \\
\hline
\end{tabular}

\section{Loading conditions:}

The two fixed capacitors, given in Table-2, though are not required at low load conditions to meet the reactive power demand, still serve to lower the reactive burden of the system and reduce the system losses from $48.78 \mathrm{~kW}$ to $38.048 \mathrm{~kW}$ in addition to improving the voltage profile. At full load condition, the lowest VSI of 0.926 before $\mathrm{CP}$ is enhanced to the safe level of 0.96 besides reducing the loses from 210.97 to $153.853 \mathrm{~kW}$ and improving the voltage profile. This performance is obtained in medium as well as over load conditions as seen from Table-3. It is therefore clear that the optimal CP enhances voltage stability, improves voltage profile and reduces the system losses.

\section{9 node system:}

The minimum and maximum reactive power demands for 69 node system are $1347 \mathrm{kVAR}$ and $2964 \mathrm{kVAR}$ respectively.
The required reactive power compensation, size and type of capacitor banks, performance before and after $\mathrm{CP}$ are given in Tables 4, 5 and 6 respectively. These results also reveal that there is significant improvement in system performance in terms of voltage stability, voltage profile and system losses.

\section{CONCLUSION}

A CP algorithm for voltage stability enhancement of radial distribution system has been developed. This method finds the optimal locations and determines the size and type of capacitor banks to be placed to enhance the voltage stability besides improving the voltage profile and reducing the system losses. The algorithm selects only one node at a time irrespective of the system size and compute $\Delta Q_{m}$ at the chosen node during the iterative process, which involves very simple computations and hence is suitable for practical implementation on systems of any size.

\section{Acknowledgements}

The authors gratefully acknowledge the authorities of Annamalai University for the facilities offered to carry out this work.

\section{REFERENCES}

1. C. W. Taylor, Power System Voltage Stability, McGraw-Hill, Inc., 1994.

2. P. Kessel and M. Glavitch, "Estimating the voltage stability of a power system", IEEE Trans. Power Delivery, 1986, PWRD-I (3), pp.346-54.

3. R. B. Prada., .L.J. Souza, "Voltage stability and thermal limit: constraints on the maximum loading of electrical energy distribution feeders", IEE Proc. Gen. Trans and Dist., 1998, Vol. 145 (5), pp. 573 77.

4. G.B.Jasmon and L.H.C.C.Lee, "Distribution network reduction for voltage stability analysis and load flow calculations", International Journal of Electrical Power and Energy Systems, 1991, Vol. 13, No. 1, pp. 9-13.

5. F. Gubina and B. Strmenik, "A simple approach to voltage stability assessment in radial networks", IEEE Trans. on Power Systems, 1997, Vol.12, No. 3, pp. 1121-28.

6. M. Chakravorty and D. Das, "Voltage stability analysis of radial distribution networks", International Journal of Electrical Power and Energy Systems, 2001, Vol. 23, No. 2, pp. 129-135.

7. M. H. Haque, "A linear voltage stability margin for radial distribution systems", Proc. IEEE PES General Meeting, Montreal, Canada, June-2006, pp. 1-6.

8. B. Venkatesh and B. Ranjan, "Fuzzy EP algorithm and dynamic data structure for optimal capacitor allocation in radial distribution systems", IEE Proc. Gen. Trans and Dist., 2006, Vol. 153, No. 5, pp. 80-88. 
9. M.-R. Haghifam and O. P. Malik, "Genetic algorithm-based approach for fixed and switchable capacitors placement in distribution systems with uncertainity and time varying loads", IET Gener. Transm. Distrib., 2007, Vol.1, No.2, pp. 244-252.

10. P. V. Prasad, S. Sivanagaraju and N. Sreenivasalu, “ A fuzzy-genetic algorithm for optimal capacitor placement in radial distribution systems", ARPN Journal of Engineering and Applied Sciences, 2007, Vol. 2, No. 3, pp. 28-32.

11. R. Srinivasa Rao and S. V. L. Narasimham, "Optimal capacitor placement in a radial distribution system using plant growth simulation algorithm", International Journal of Electrical Power and Energy Systems Engineering, 2008, Vol. 1, No. 2, pp. 123-130.

12. H. M. Khodr, F. G. Olsina, P. M. De Oliveira-De Jesus and J. M. Yusta, "Maximum savings approach for location and sizing of capacitors in distribution systems", Electric Power System Research, 2008, Vol. 78, No. 4, pp. 1192-1203.

13. Amgad A. EL-Dib, Hosam K.M. Youssef, M.M. EL-Metwally and Z. Osman, Optimum VAR sizing and allocation using particle swarm optimisation", Electric Power System Research, 2008, Vol. 77, No. 8, pp. 965-972.

14. J.C. Hernandez, A. Medina and F. Jurado, "Optimal allocation and sizing for profitability and voltage enhancement of PV systems on feeders", Renewable Energy Journal, 2007, Vol. 32, No. 10, pp. 1768-1789.

15. N.G.A. Hemda and M. Kurrat, "Distributed generation location and capacity effect on voltage stability of distribution networks, Annual IEEE Conference, 2008, pp. 1-5

16. M. El Arini, "Optimal capacitor placement incorporating voltage stability and system security" ETEP, 2000, Vol. 10, No. 5, pp.319-325.

17. P. K. Satpathy, D. Das and P. B. Dutta Gupta, "Critical switching of capacitors to prevent voltage collapse", Electric Power Systems Research, 2004, Vol. 71, No. 1, pp. 11-20.
18. B. Jasmon and L. H. C. C. Lee, "Maximising voltage stability in distribution networks via loss minimisation", Journal of Electrical Power and Energy Systems, 1991, Vol. 13, No. 3, pp. 148-152.

19. M. A. Kashem and M. Moghaavvemi, "Maximising radial voltage stability and load balancing via loss minimisation in distribution networks", Proceedings of Energy Management and Power Delivery, March 1998, Vol. 1, pp. 91-96.

20. J. C. Wang, H. C. Chiang and G. R. Darling, "An efficient algorithm for real-time network reconfiguration in large scale unbalanced distribution systems", IEEE Trans. on Power Systems, 1996, Vol.11, No. 1, pp. 511-17.

21. M. A. Kashem, V. Ganapathy and G.B.Jasmon, “ Network reconfiguration for enhancement of voltage stability in distribution networks", IEE Proc. Gen. Trans and Dist., 2000, Vol. 147 (3), pp. 171-75

22. S. Sivanagaraju, N. Visali, V. Sankar and T. Ramana, " Enhancing voltage stability of radial distribution systems by network reconfiguration", Electric power components and systems, 2004, Vol.33, No. 5, pp. 539-50.

23. N.C.Sahoo and K. Prasad, "A fuzzy genetic approach for network reconfiguration to enhance voltage stability in radial distribution systems", Energy Conversion and Management, 2006, Vol.47, No. 18 , pp. 3288-306.

24. B. Venkatesh and R. Ranjan, "Optimal radial distribution system reconfiguration using fuzzy adaptation of evolutionary programming", Journal of Electrical Power and Energy Systems, 2003, Vol. 25 , No. 10 , pp. $775-780$.

25. P. Aravindhababu, S. Ganapathy and K. R. Nayar, "A novel technique for the analysis of radial distribution systems", International Journal of Electrical Power and Energy Systems, 2001, Vol. 23, No. 3, pp. 167-171. 